\title{
Diagnostic accuracy of contrast enhanced MRI pelvis in differentiating indeterminate adnexal lesions into benign and malignant with histopathological correlation
}

\author{
Sorath Murtaza ${ }^{1}$, Junaid Iqbal ${ }^{1 *}$, Saad Ahmed ${ }^{1}$, Ashraf Amir Ali ${ }^{1}$, \\ Marya Hameed ${ }^{2}$, Muhammad Kashif Shazlee ${ }^{3}$
}

\begin{abstract}
${ }^{1}$ Department of Radiology, Dr. Ziauddin University Hospital, Karachi, Pakistan
${ }^{2}$ Department of Radiology, National Institute of Child Health, Karachi, Pakistan

${ }^{3}$ Department of Radiology, The Indus Hospital, Karachi, Pakistan
\end{abstract}

Received: 05 May 2021

Accepted: 29 May 2021

*Correspondence:

Dr. Junaid Iqbal,

Email: junaid2008@gmail.com

Copyright: (c) the author(s), publisher and licensee Medip Academy. This is an open-access article distributed under the terms of the Creative Commons Attribution Non-Commercial License, which permits unrestricted non-commercial use, distribution, and reproduction in any medium, provided the original work is properly cited.

\begin{abstract}
Background: The objective of our study was to determine the diagnostic accuracy of contrast-enhanced magnetic resonance imaging pelvis in differentiating indeterminate adnexal lesions into benign and malignant, while considering histopathological examinations as the gold standard.

Methods: A total 880 patients who underwent contrast enhanced MRI pelvis in our institute from January 2014 to June 2016 were prospectively analyzed.

Results: A total of 880 women were included in this study, of which $782(88.8 \%)$ were younger than 50 years and 98 $(11.1 \%)$ were older than 50 years. Mean patient age was 56.7 years and mean tumor size was $4.38 \mathrm{~cm}$. There were $648(73.60 \%)$ patients who had a tumor size of $>4 \mathrm{~cm}$, and $337(38.29 \%)$ of these tumors were found to be malignant. Furthermore, tumors smaller than $4 \mathrm{~cm}$ in size were observed in $232(26.36 \%)$ of patients, of which tumors in 225 $(25.56 \%)$ patients were benign.

Conclusions: The diagnostic accuracy of contrast-enhanced MRI was found to be significantly high (79.65\%) in differentiating indeterminate adnexal lesions into benign and malignant lesions.
\end{abstract}

Keywords: Contrast-enhanced MRI, Ovarian tumor, Benign and malignant, Histopathology

\section{INTRODUCTION}

Ovarian cancer is the sixth most common cancer in women and seventh most common cause of cancer deaths. ${ }^{1}$ In Pakistan, ovarian cancer is the fourth most common cancer in women. Overall, it accounts for $4 \%$ of all cancers in females. ${ }^{2}$ The lifetime risk of dying from invasive ovarian cancer is approximately 1 in 95 . If diagnosed at an early stage - stage I, i.e., confined to one or two ovaries, there is a $>90 \%$ survival rate at 5 years. $^{3}$ Typically most ovarian tumors arise from the epithelium, and these tumors account for $60 \%$ of all ovarian tumors and $85 \%$ of all malignant ovarian tumors. The most common tumors are serous and mucinous cystadenocarcinoma. Other less common varieties include clear cell carcinoma and endometroid carcinoma. ${ }^{4}$ It is a diagnostic dilemma when imaging findings of ovarian lesion cannot be categorized benign or malignant. Currently, ultrasonography (US), computed tomography (CT), and magnetic resonance imaging (MRI) are used to evaluate ovarian lesions. US is the first-line imaging investigation to detect and characterize ovarian masses. ${ }^{5}$ An ovarian mass is categorized as indeterminate on US when it cannot be confidently categorized as benign or malignant, despite conducting through examinations using Doppler assessment. Alternatively an ovarian mass 
for which the site of origin, from the ovary, uterus, or another pelvic structure, remains to be established is categorized as indeterminate. US is the primary imaging modality for adnexal masses, however, MRI has shown to be the most effective mode of imaging, especially for categorizing borderline and indeterminate masses. Presence of hemorrhage, fat, and collagen can be accurately identified with MRI. ${ }^{6}$ MRI has an overall accuracy of $88-93 \%$ in distinguishing benign from malignant ovarian tumors. ${ }^{7} \mathrm{MRI}$ is useful for definitively diagnosing many common benign adnexal lesions. MRI better characterizes indeterminate adnexal lesions visualized via US. This is particularly evident in cases when an extraovarian cystic lesion is suspected but a normal ipsilateral ovary is not seen and when a predominantly solid lesion requires more tissue-specific characterization for diagnosis. Cystic extraovarian lesions include peritoneal inclusion cysts, paratubal cysts, and hydrosalpinx. Solid-appearing adnexal lesions include dermoids, exophytic uterine and broad ligament fibroids and ovarian fibrothecomas. ${ }^{8}$

\section{Objective}

Current study aimed to determine the diagnostic accuracy of contrast-enhanced MRI pelvis in differentiating indeterminate adnexal lesions into benign and malignant with histopathological correlation.

\section{METHODS}

A cross-sectional prospective study was conducted with 880 patients. The inclusion criteria of this study were women who presented with a complex adnexal mass lesion on US with thick walls, septations, a solid mural component and flow on color Doppler, patients with raised CA-125 levels (>35 IU/ml) and postmenopausal women with vague abdominal pain for $>6$ months. Risk factors such as nulliparity, early menarche, and late menopause were also assessed. We excluded patients who were already diagnosed as ovarian tumor on histopathology, were already diagnosed and were on treatment, showed recurrence of disease after treatment, have some contraindications for contrast (gadolinium) administration, i.e., hypersensitivity, renal failure or pregnancy and were not fit for surgery.

An informed consent was obtained from all patients after the purpose, procedure, and risk-benefits ratio were explained to them. MRI pelvis with contrast was performed on a $1.5 \mathrm{~T}$ MRI machine with a $3-4 \mathrm{~mm}$ slice thickness and a reconstruction interval of $1 \mathrm{~mm}$ extending from the lower lumbar to the lower margins of pubic symphysis. Axial sections were taken in plain and after injections of $0.2 \mathrm{ml} / \mathrm{kg}$ of gadolinium contrast via a power injector. Sagittal and coronal images were also acquired. Magnetic resonance images were analyzed by two senior consultant radiologists working independently in a radiology department for a minimum of 7 years. Ovarian tumors were analyzed on the basis of their size and appearance parameters such as solid cum cystic lesions, purely cystic lesions, thick septations, lobulated solid mass showing tumor vessels on contrast-enhanced images, and enhancing papillary projections. Furthermore, we assessed the extent of the disease, i.e., the presence of lymphadenopathy, involvement of both ovaries or nearby soft tissues, ascites, omental caking, and deposits, was also assessed. A pro forma was used to document the findings after MRI imaging. After surgical evacuation, specimen was sent to the laboratory for histopathological examinations. The final diagnosis depended on histopathological findings, which were taken as the gold standard for this study.

Data compilation and statistical analyses were done on SPSS version 16. Descriptive statistics, frequency, and percentage were computed for presentation of qualitative variables including MRI and histopathological findings. Age and size of the tumor were presented as mean \pm standard deviation. A $2 \times 2$ table was constructed. Sensitivity, specificity, positive and negative predictive values, and diagnostic accuracy of MRI pelvis were determined by considering histopathological examination as the diagnostic gold standard. Patient data were stratified according to the age and size of the tumor, and parity was applied to control the effect modifier by applying the chi-square test and statistical significance was indicated by $\mathrm{p} \leq 0.05$.

\section{RESULTS}

Among the 880 women included in the study, 782 $(88.8 \%)$ were younger than 50 years and $98(11.1 \%)$ were older than 50 years. The mean age of the patients was 56.7 years, and the mean size of the tumor was $4.38 \mathrm{~cm}$. There were $648(73.60 \%)$ patients with tumor size $>4 \mathrm{~cm}$, and $337(38.29 \%)$ of these tumors were found to be malignant. Of the patients, $232(26.36 \%)$ had tumor size of $<4 \mathrm{~cm}$, in which $225(25.56 \%)$ of these tumors were benign. Nulliparity was found in $384(43.60 \%)$ patients, whereas multiparity was found in $496(56.40 \%)$ patients.

The primary lesion was classified into three categories. Most of the lesions were solid cum cystic (39.54\%), followed by purely cystic lesions $(32.84 \%)$ and lobulated solid lesions $(27.61 \%)$. Thick septations were present in $40.45 \%$ whereas enhancing papillary projections were present in $44.2 \%$ of the cases. Unilateral involvement was noted in $63.29 \%$ of the cases whereas bilateral involvement was observed in $36.7 \%$ of the cases. In addition, secondary signs were also assessed. Pelvic lymphadenopathy was identified in $38.75 \%$ of the cases, ascites in $37.0 \%$ and omental deposits in $14.5 \%$ (Table 1).

On the basis of MRI findings, benign and malignant ovarian lesions were diagnosed in 263 and 617 of the patients respectively. Of the 263 patients diagnosed with benign lesions, histopathological examination revealed that the tumors in 214 were benign, however, there were 
49 false negative histopathological outcomes showing malignancy. Of the 617 patients labelled as having malignant lesions, 487 were also positive on histopathology but 130 patients had false positive results for the presence of malignancy (Table 2). The sensitivity and specificity of contrast-enhanced MRI pelvis in differentiating indeterminate ovarian lesions were found to be $90.85 \%$ and $62.20 \%$ respectively. The overall accuracy was $79.65 \%$ for differentiating benign from malignant ovarian lesions. The positive and negative predictive values were $78.93 \%$ and $81.36 \%$, respectively. The Chi-square test was applied and the outcome was statistically significant $(\mathrm{p}<0.001)$ (Table 3$)$.

Table 1: MRI criteria for benign and suspicious adnexal lesions.

\begin{tabular}{|lll|}
\hline Criterias & Benign & Malignant \\
\hline Principal criteria & $<4$ & $>4$ \\
\hline Size $(\mathrm{cm})$ & No & $\begin{array}{l}\text { Solid part of the mass with heterogeneous } \\
\text { enhancement pattern }\end{array}$ \\
\hline Solid components & Simple & With vegetation and internal structures \\
\hline Cystic mass & $<3$ & $>3$ \\
\hline Thickness of wall or septa $(\mathrm{mm})$ & No & Yes \\
\hline Lobulated mass & Wall of cyst, dense & Tiny amorphic \\
\hline Calcifications & No & Yes \\
\hline Necrosis & No & Yes, with heterogeneous enhancement pattern \\
\hline Papillary projections & No & Yes, with heterogeneous enhancement pattern \\
\hline Tumor vessels & & \\
\hline Additional criteria & Normal (short axis $<1 \mathrm{~cm})$ & Enlarged (short axis $>1 \mathrm{~cm})$ \\
\hline Lymph nodes & No & Yes \\
\hline Peritoneal implants & & \\
\hline
\end{tabular}

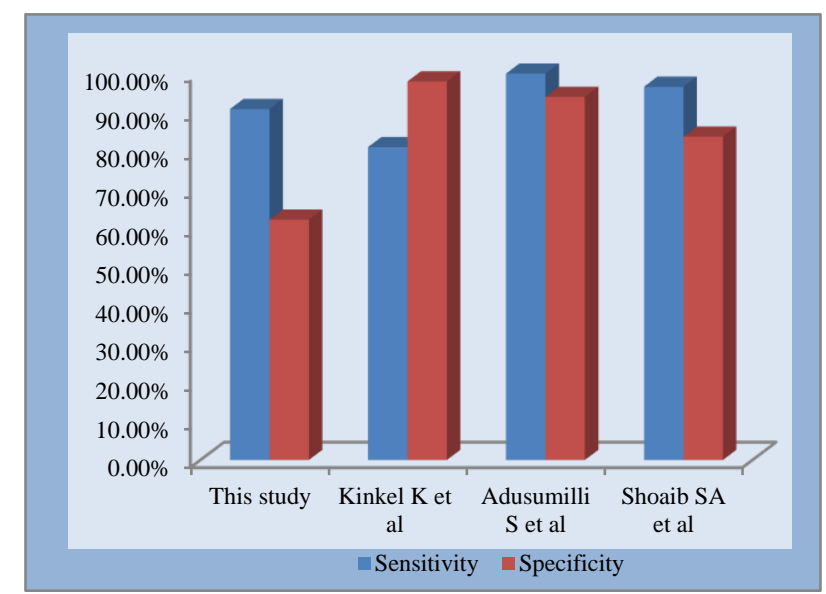

Figure 1: Comparison of sensitivity and specificity with different studies.

\section{DISCUSSION}

This study was a prospective study of 880 patients who were clinically suspected for for the presence of indeterminate adnexal lesions. The study included women who presented with a complex indeterminate adnexal mass lesion on US with thick walls, septations, a solid mural component, and flow on color Doppler, patients with raised CA-125 levels (>35 IU/ml), and postmenopausal women with vague abdominal pain for more than 6 months. Most of the women were younger than 50 years (mean age 56.7 years). Nulliparity was found in 384 (43.60\%) patients whereas multiparity was found in $496(56.40 \%)$ patients. The most important diagnostic criteria for ovarian malignancy include lesion larger than $4 \mathrm{~cm}$ in size, thickness of walls and septa of $>3 \mathrm{~cm}$, presence of papillary projections and a solid mural component, nodularity, necrosis, and hemorrhage (Table 4). Although contrast enhancement usually increases the accuracy in differentiation of benign and malignant lesions, its usefulness may be limited for some types of tumors. ${ }^{9}$

In this study, $73.60 \%$ patients had tumor size of $>4 \mathrm{~cm}$ and $26.36 \%$ of the patients had tumor size of $<4 \mathrm{~cm}$. Most of the tumors that were $>4 \mathrm{~cm}$ in size $(38.29 \%)$ were found to be malignant. However, $25.56 \%$ of the tumors that were $<4 \mathrm{~cm}$ in size were benign. Most of the lesions were solid cum cystic (39.54\%), followed by purely cystic lesions (32.84\%) and lobulated solid lesions $(27.61 \%)$. Notable, when a cystic adnexal mass can be shown to be separate from the ipsilateral ovary (extraovarian), it is typically benign. 8,9

Papillary projections are specific features of epithelial ovarian neoplasms. Similar to ovarian cancer, a cystadenoma may display papillary projections, although it does so less frequently (9\%) than malignant tumors. Papillary projections enhance after contrast administration, facilitating differentiation from an intracystic clot or debris. ${ }^{10}$ In current study, thick septations were present in $40.45 \%$ of the cases, whereas enhancing papillary projections were present in $44.2 \%$. 
Unilateral and bilateral involvement was observed in $63.29 \%$ and $36.7 \%$ of the cases respectively. Ancillary criteria that serve to definitely characterize a tumor as malignant include involvement of pelvic organs or sidewall; peritoneal, mesenteric, or omental disease; ascites; and adenopathy. When these criteria are used, the sensitivities and specificities for malignancy range between $91 \%$ and $92 \%$ and between $91 \%$ and $100 \%$ respectively. ${ }^{9,11} \mathrm{We}$ found pelvic lymphadenopathy in $38.75 \%$ of the cases, ascites in $37.0 \%$ and omental deposits in $14.5 \%$.

Table 2: Summary of results according to diagnostic criteria for ovarian lesions on MRI Pelvis.

\begin{tabular}{|ll|}
\hline Parameters & $\%$ \\
\hline Primary lesion & \\
\hline Purely cystic & 32.84 \\
\hline Solid cum cystic & 39.54 \\
\hline Lobulated solid & 27.61 \\
\hline Thick septations & 40.45 \\
\hline Papillary projections & 44.20 \\
\hline Site of involvement & \\
\hline Unilateral & 63.29 \\
\hline Bilateral & 36.70 \\
\hline Size (cm) & \\
\hline$<4$ & 26.36 \\
\hline$>4$ & 73.60 \\
\hline Secondary signs & \\
\hline Ascites & 37.00 \\
\hline Pelvic lymphadenopathy & 38.75 \\
\hline Omental deposits & 14.50 \\
\hline Unilateral & 63.29 \\
\hline
\end{tabular}

This study concludes that the sensitivity, specificity, and diagnostic accuracy of contrast enhanced MRI pelvis in differentiating indeterminate ovarian lesions were $90.85 \%, 62.20 \%$ and $79.65 \%$ respectively. The positive and negative predictive values were $78.93 \%$ and $81.36 \%$ respectively. A meta-analysis evaluating the incremental value of a second test for an indeterminate adnexal mass detected on gray scale US by Kinkel et al determined that MRI with IV contrast administration provided the highest posttest probability of ovarian cancer when compared with CT, Doppler US, or MRI without contrast. ${ }^{12}$ The sensitivity and specificity of unenhanced MRI was $76 \%$ and $96 \%$ respectively, and those of enhanced MRI were $81 \%$ and $98 \%$ respectively. The sensitivity and specificity of Doppler US were $82 \%$ and $84 \%$ respectively. In another prospective series by Adusumilli et al contrastenhanced MRI showed a sensitivity and specificity of $100 \%$ and $94 \%$ respectively, in the diagnosis of ovarian malignancy. ${ }^{13}$ In a prospective study by Sohaib et al of women with suspected adnexal masses, both Doppler US and MRI were highly sensitive for identifying malignant lesions (US, 100\%, MRI, 96.6\%), but the specificity of MRI was significantly greater (US, 39.5\%, MRI, 83.7\%) (Figure 1). ${ }^{14}$
Ovarian masses present a special diagnostic challenge when imaging findings cannot be categorized into benign or malignant pathology. US, CT and MRI are currently used to evaluate ovarian tumors. The goal of imaging in ovarian cancer detection is to expeditiously distinguish benign adnexal lesions from those requiring further pathologic evaluation for malignancy. For lesions that are indeterminate on US, contrast-enhanced MRI increases the specificity of imaging evaluation.

\section{CONCLUSION}

The diagnostic accuracy of contrast-enhance MRI was found to be significantly high $(79.65 \%)$ in differentiating indeterminate adnexal lesions into benign and malignant.

Funding: No funding sources

Conflict of interest: None declared

Ethical approval: The study was approved by the Institutional Ethics Committee

\section{REFERENCES}

1. Ferlay J, Bray F, Pisani P, Parkin DM. Available at: https://gco.iarc.fr/. Accessed on 20 February 2021.

2. Malik IA. A prospective study of clinic-pathological features of epithelial ovarian cancer in Pakistan. J Pak Med Assoc. 2002;52(4):155-8.

3. Horner MJ, Ries LAG, Krapcho M. SEER cancer statistics review. Available at: https://seer.cancer.gov/ csr/1975_2018/. Accessed on 20 February 2021.

4. Kim MY, Rha SE, Oh SN. MR Imaging findings of hydrosalpinx: a comprehensive review. Radiographics. 2009;29:495-507.

5. Pierce N, Narayanan P, Sahdev A. Ovarian lesions pose diagnostic dilemmas. Diagn Imaging Eur. 2008;24(3):14-8.

6. Spencer JA, Forstner R, Cunha TM. ESUR guidelines for MR imaging of the sonographically indeterminate adnexal mass: an algorithmic approach. Eur Radiol. 2010;20(1):25-35.

7. Bazot M, Daraï E, Nassar-Slaba J. Value of magnetic resonance imaging for the diagnosis of ovarian tumors: a review. J Comput Assist Tomogr. 2008; 32(5):712-23.

8. Iyer VR, Lee SI. MRI, CT, and PET/CT for ovarian detection and adnexal lesion characterization. Am J Roentgenol. 2010;194:311-21.

9. Stevens SK, Hricak H, Stern JL. Ovarian lesions: detection and characterization with gadolinium enhanced MR imaging at $1.5 \mathrm{~T}$. Radiology. 1991;181:481-8.

10. Ghossian MA, Buy JN, Ligneres C. Epithelial tumors of the ovary: comparison of MR and CT findings. Radiology. 1991;181:863-70.

11. Hircak H, Chen M, Coakley FV. Complex adnexal masses: detection and characterization with MR imaging-multivariate analysis. Radiology. 2000;214: $39-46$. 
12. Kinkel K, Lu Y, Mehdizade A. Indeterminate ovarian mass at US: incremental value of second imaging test for characterization-meta-analysis and Bayesian analysis. Radiology 2005; 236:85-94.

13. Adusumilli S, Hussain HK, Caolil EM. MRI of sonographically indeterminate adnexal masses. AJR. 2006;187:732-40.

14. Sohaib SA, Mills TD, Sahdev A. The role of magnetic resonance imaging and ultrasound in patients with adnexal masses. Clin Radiol. 2005;60:340-8.
Cite this article as: Murtaza S, Iqbal J, Ahmed S, Ali AA, Hameed M, Shazlee MK. Diagnostic accuracy of contrast enhanced MRI pelvis in differentiating indeterminate adnexal lesions into benign and malignant with histopathological correlation. Int $\mathbf{J}$ Basic Clin Pharmacol2021;10:846-50. 